\title{
Video Article \\ Intrathoracic Injection for the Study of Adult Zebrafish Heart
}

\author{
Thomas Bise ${ }^{1}$, Anna Jaźwińska \\ ${ }^{1}$ Department of Biology, University of Fribourg
}

Correspondence to: Anna Jaźwińska at anna.jazwinska@unifr.ch

URL: https://www.jove.com/video/59724

DOI: doi:10.3791/59724

Keywords: Biology, Issue 147, intrathoracic injection, microinjection, adult zebrafish, heart regeneration, cardiac preconditioning, heart stimulation, epicardium

Date Published: 5/14/2019

Citation: Bise, T., Jaźwińska, A. Intrathoracic Injection for the Study of Adult Zebrafish Heart. J. Vis. Exp. (147), e59724, doi:10.3791/59724 (2019).

\section{Abstract}

The adult zebrafish heart provides a powerful model in cardiac regeneration research. Although the strength of this system is based on transgenic approaches, a rapid delivery of exogenous factors provides a complementary technique in functional studies. Here, we present a method that relies on administration of a few microliters of solution into the pericardial cavity without causing myocardial damage. Intrathoracic (IT) injections can efficiently deliver proteins and chemical compounds directly onto the heart surface. The injected substances diffuse through the epicardium into the underlying cardiac tissues. Compared to intraperitoneal (IP) injections, the main advantage of intrathoracic injections is the focal administration of the tested factors on the target organ. The delivery of molecules directly into the pericardium is a suitable strategy for studies of cardiac preconditioning and regeneration in adult zebrafish.

\section{Video Link}

The video component of this article can be found at https://www.jove.com/video/59724/

\section{Introduction}

Among vertebrates, zebrafish possess a remarkable capacity to regenerate their hearts ${ }^{1,2}$. This ability has been reported in several injury models, namely ventricular apex resection, cryoinjury $(\mathrm{Cl})$ and genetic cardiomyocyte ablation ${ }^{3,4,5,6,7}$. After invasive injuries, the damaged wall of the ventricle becomes transiently healed by fibrotic tissue, which is progressively replaced by a new myocardium ${ }^{8,9,10,11}$. The early wound healing response involves epicardium activation and recruitment of immune cells ${ }^{12,13,14,15}$. Concomitantly, cardiomyocytes near the injured myocardium become activated, dedifferentiate, proliferate and progressively replace the wounded area within 30-90 days ${ }^{16,17,18,19}$. Substantial progress in deciphering the molecular and cellular mechanisms of cardiac regeneration has been achieved thanks to the availability of genetic tools, such as cell-lineage tracing analysis, inducible gene overexpression, fluorescent tissue reporter lines, and CRISPR/Cas 9 gene mutagenesis ${ }^{20,21}$.

We have recently established a model of cardiac preconditioning in the adult zebrafish by thoracotomy ${ }^{22,23}$. Preconditioning increases expression of cardioprotective genes and elevates the re-entry into the cell cycle in the intact and regenerating hearts. These processes are associated with the recruitment of immune cells and matrix remodeling ${ }^{22,24}$. The mechanisms of preconditioning are poorly understood, and the establishment of new techniques is required to foster this area of research. In particular, optimized administration of secreted signaling proteins or other chemical compounds is essential to further investigate this topic.

Being aquatic animals, zebrafish can naturally absorb various substances dissolved in water through their gills and skin. This offers a possibility for non-invasive drug delivery through immersion of fish in solutions with diverse chemicals, such as pharmacological inhibitors, steroid hormones, tamoxifen, BrdU and antibiotics. Indeed, numerous studies from various laboratories, including ours ${ }^{25,26,27}$, have taken advantage of this method, which is particularly valuable in the field of regenerative biology ${ }^{6,28}$. This approach is, however, not appropriate for delivery of peptides, DNA, RNA, morpholinos or molecules with a limited tissue permeability. In these cases, a more efficient delivery is achieved by microinjection into the body, for example, by inserting the capillary into the retro-orbital venous sinus, into the intraperitoneal or intrapericardial cavity $^{29,30,31}$. Here, we describe a procedure of intrathoracic injection of a small amount of solution, as a suitable method to study heart regeneration and preconditioning in adult zebrafish.

\section{Protocol}

Animal care and all animal procedures described in the following protocol were approved by the cantonal veterinary office of Fribourg, Switzerland.

\section{Tools and Solutions for Injections}

1. Pull microinjection-adapted borosilicate glass capillaries using a needle puller according to Figure $\mathbf{1 A}$. Store pulled capillaries in a $9 \mathrm{~cm}$ Petri dish with rails of modeling clay or adhesive tape.

2. Using common scissors, cut a piece of sponge $(7 \mathrm{~cm} \times 3 \mathrm{~cm} \times 1 \mathrm{~cm})$ and carve a fish-like silhouette in its middle. 
3. Prepare small aliquots of injection solution with the tested proteins or other compounds. Adjust their concentration dependently on the assay by diluting the substance in 1x Hanks balanced salt solution (HBSS) complemented with $10 \%$ phenol red.

NOTE: Here, the concentration of the tested protein was $100 \mathrm{ng} / \mathrm{mL}$.

4. To prepare a stock solution of buffered tricaine anesthetics, dissolve $4 \mathrm{~g}$ of tricaine in $980 \mathrm{~mL}$ of distilled water. Adjust pH to $7.0-7.4$ using $1 \mathrm{M}$ Tris- $\mathrm{HCl} \mathrm{pH} \mathrm{9,} \mathrm{and} \mathrm{fill} \mathrm{up} \mathrm{with} \mathrm{water} \mathrm{to} 1,000 \mathrm{~mL}$. Store the solution in dark at $4{ }^{\circ} \mathrm{C}$.

5. To obtain the working concentration of anesthetics, add 1-2 $\mathrm{mL}$ of tricaine stock solution into $50 \mathrm{~mL}$ of fish water in a beaker. NOTE: The working concentration of tricaine anesthetics should be prepared freshly before use.

\section{Preparation of the Injection Station}

1. Turn on the stereomicroscope with the light from the top and adjust magnification to 16x.

2. Soak the sponge with fish water, place it on a $9 \mathrm{~cm}$ Petri dish on the microscope stage and adjust focus.

3. Under the stereomicroscope, cut the end of a microcapillary at $\sim 7 \mathrm{~mm}$ from the basis using an iridectomy scissors as shown in Figure $1 \mathrm{~A}$. The ideal tip diameter would be $\sim 20 \mu \mathrm{m}$.

NOTE: Cutting the tip of the capillary in an oblique way is optimal for insertions into the tissue.

4. Insert the microcapillary into the needle holder of the microinjector apparatus.

5. Using microloader tips, load a control solution (e.g., 1x HBSS) to set up the pressure of injection, in order to obtain the appropriate flow ranging between $0.3 \mu \mathrm{L} / \mathrm{s}$ and $0.5 \mu \mathrm{L} / \mathrm{s}$. Empty the needle.

6. Load the selected volume of the injection solution (e.g., ciliary neurotrophic factor [CNTF] diluted in $1 \times$ HBSS) into the tip of the capillary (Figure 1B). There should be no air bubble in the capillary.

NOTE: The maximal volume of injection solution depends on the fish size. For a standard length of $2.5-3 \mathrm{~cm}$ (distance from the snout to the caudal peduncle), the maximum injection volume that prevents exessive thoracic swelling and bleeding was determined to be $5 \mu \mathrm{L}$ (Figure 1F). Larger volumes could be injected to bigger fish.

\section{Preparation of the Fish for Intrathoracic Injection}

1. Catch an adult zebrafish (Danio rerio) with a net and transfer it into the anesthetic solution.

2. After 1-2 min, when fish stops swimming and the movement of operculum is reduced, touch the fish with a plastic spoon to make sure it does not react to any contact.

3. Quickly and carefully transfer the fish with the spoon into the groove of the wet sponge, with ventral side up. The head of the fish should point away from the operator's dominant-hand.

\section{Microinjection into the Pericardium}

1. Under the stereomicroscope, carefully observe the movement of the beating heart under the skin of the fish. Visually determine the injection point above the beating heart and in the middle of the triangle defined by the ventral cartilaginous plates (Figure 1D). Insert the tip of the capillary at $30-45^{\circ}$ degree angle relative to the body axis (Figure 1E). Gently penetrate the skin with the tip of the microcapillary into the pericardium (Figure 1C). An optimal entry point is closer to the abdomen than to the head.

NOTE: Do not insert the capillary too deeply into the body and the heart, as this will cause injury to the organ. In case of heart puncture, the needle generally fills with blood. If this happens, remove the capillary and exclude the fish from the experiment.

2. Once the needle is inside the pericardium, complete injection by pressing the pedal of the microinjector device.

NOTE: Be careful not to inject air into the thoracic cavity.

3. After injection, gently withdraw the capillary from the thorax and immediately transfer the fish into a tank with system water for recovery.

4. Monitor the fish until total recovery from anesthesia.

5. Collect heart at the desired time point and prepare it for further analysis.

NOTE: In case the fish does not resume movement of the operculum within $30 \mathrm{~s}$, reanimate the fish by squeezing water into the gills with a plastic pipette.

\section{Representative Results}

After intrathoracic (IT) injections, the effects of exogenous solution can be analyzed. For this purpose, the fish should be euthanized and the hearts collected, fixed and histologically processed, according to previously published protocols ${ }^{32,33}$.

To validate the method, we first performed two test experiments by injecting color and fluorescent dyes. First, we euthanized fish and postmortem injected $3 \mu \mathrm{L}$ of ink into the thorax. The hearts were collected after $5 \mathrm{~min}$, washed in phosphate-buffered saline (PBS), fixed in $2 \%$ formalin, washed in PBS and photographed under the microscope. Second, we injected $3 \mu \mathrm{L}$ of $1 \mu \mathrm{g} / \mathrm{mL} 4^{\prime}, 6$-diamidino-2-phenylindole (DAPI) in vivo, and fixed the heart after $2 \mathrm{~h}$. In both assays, whole-mount analysis revealed labeling of the entire heart including the ventricle, the atrium and the bulbus arteriosus (Figure 2A,B). These results reveal efficient spreading of the injected solution on the heart surface.

A common protocol for delivery of exogenous substances into the adult fish is intraperitoneal (IP) injection. To compare the suitability of IT versus IP injections for heart studies, we injected a similar amount of DAPI using both methods and fixed the hearts after 5 min and 120 min (Figure 3A). The hearts were sectioned and stained with phalloidin Alexa Fluor (AF) 568 that labels F-actin in cardiac muscle. No DAPI-positive cells were observed in the hearts after IP injection at both time points (Figure 3B). By contrast, IT injection resulted in the presence of DAPIlabeled nuclei in the myocardium (Figure 3B). These results demonstrate that IT injection improved the delivery of the compound to the heart, as compared to the IP injection.

To test the suitability of this method for heart regeneration studies, we cryoinjured ventricles ${ }^{8}$, and performed IT injections of $3 \mu \mathrm{L}$ of $1 \mu \mathrm{g} / \mathrm{mL}$ DAPI and $1 \mu \mathrm{g} / \mathrm{mL}$ phalloidin AF649 at 3 and 7 days post-cryoinjury (dpci) (Figure 4A). At $1 \mathrm{~h}$ post-injection, the hearts were collected, fixed, 
sectioned and stained with phalloidin AF568 to visualize the intact myocardium. We found that both the myocardium and the injured tissue contained numerous DAPI positive cells, indicating an efficient penetration of this dye into the intact heart and fibrotic tissue (Figure 4B). Furthermore, injected phalloidin AF649 was also incorporated by cardiomyocytes of the peri-injury zone and some recruited fibroblasts of the wounded area. This experiment reveals that the drugs can cross the epicardium and penetrate into the underlying myocardium.

After testing the efficiency of IT injections using dyes, we analyzed the effects of injected proteins on the heart. We synthetized a cytokine, called CNTF, which is upregulated after thoracotomy ${ }^{24}$. We investigated the effects of the exogenous CNTF on various processes, namely cardiomyocyte proliferation, extracellular matrix deposition, immune cell recruitment and cardioprotective gene expression. We found that all these biological aspects were activated by IT injection of CNTF, as compared to control immunoglobulins (Figure 5) ${ }^{24}$. These results demonstrate that the method of intrathoracic injection provides a suitable strategy for targeted delivery of proteins to study their effects on distinct heart tissues in a variety of assays. 


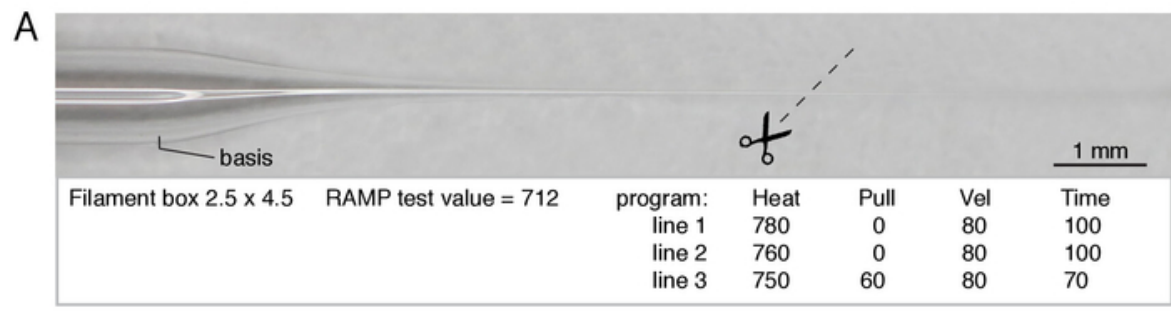

$\mathrm{B}$
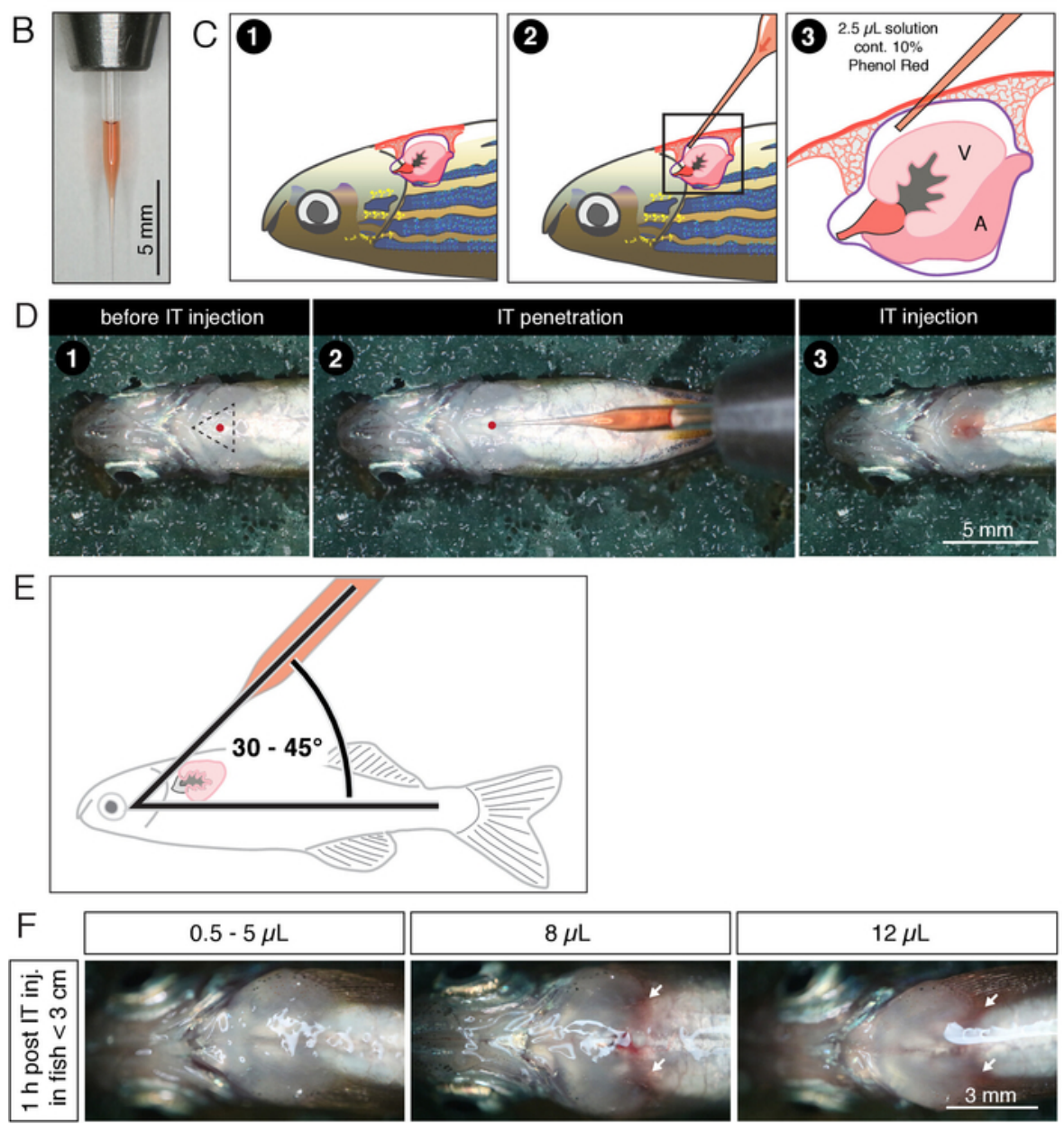

Figure 1: Intrathorascic (IT) injection in adult zebrafish. (A) Photograph of a pulled microinjection capillary with filament (6", $1.0 \mathrm{~mm}$ in diameter) and values of the needle puller program used. (B) Photograph of a pulled microinjection capillary with filament (6", $1.0 \mathrm{~mm}$ in diameter) filled up with $2.5 \mu \mathrm{L}$ of solution containing $10 \%$ phenol red. The pulled tip of the needle is maximally $7 \mathrm{~mm}$ long. (C) Schematic representation of the IT injection procedure. (D) Photographs of the IT injection procedure. This figure has been modified from Bise et al. ${ }^{24}$. Numbers in panels $\mathrm{C}$ and D correspond to the same steps of the procedure: (1) fish is placed ventral side up on a humidified sponge. The puncture site (red dot in the triangle) is located in the center of the chest near the gills. (2) Penetration of the needle into the pericardium. Red dot indicates puncture site. (3) Injection is monitored by observing the spread of the red solution in the pericardial cavity. (E) Scheme of IT injection. Angle between the injection capillary and the body axis should be between $30^{\circ}$ and $45^{\circ}$ to avoid heart puncture. (F) Photographs of fish thorax at 1 hour after IT injection of indicated volumes. White arrows are pointing at the redish tissue, which might indicate internal bleeding. Please click here to view a larger version of this figure. 

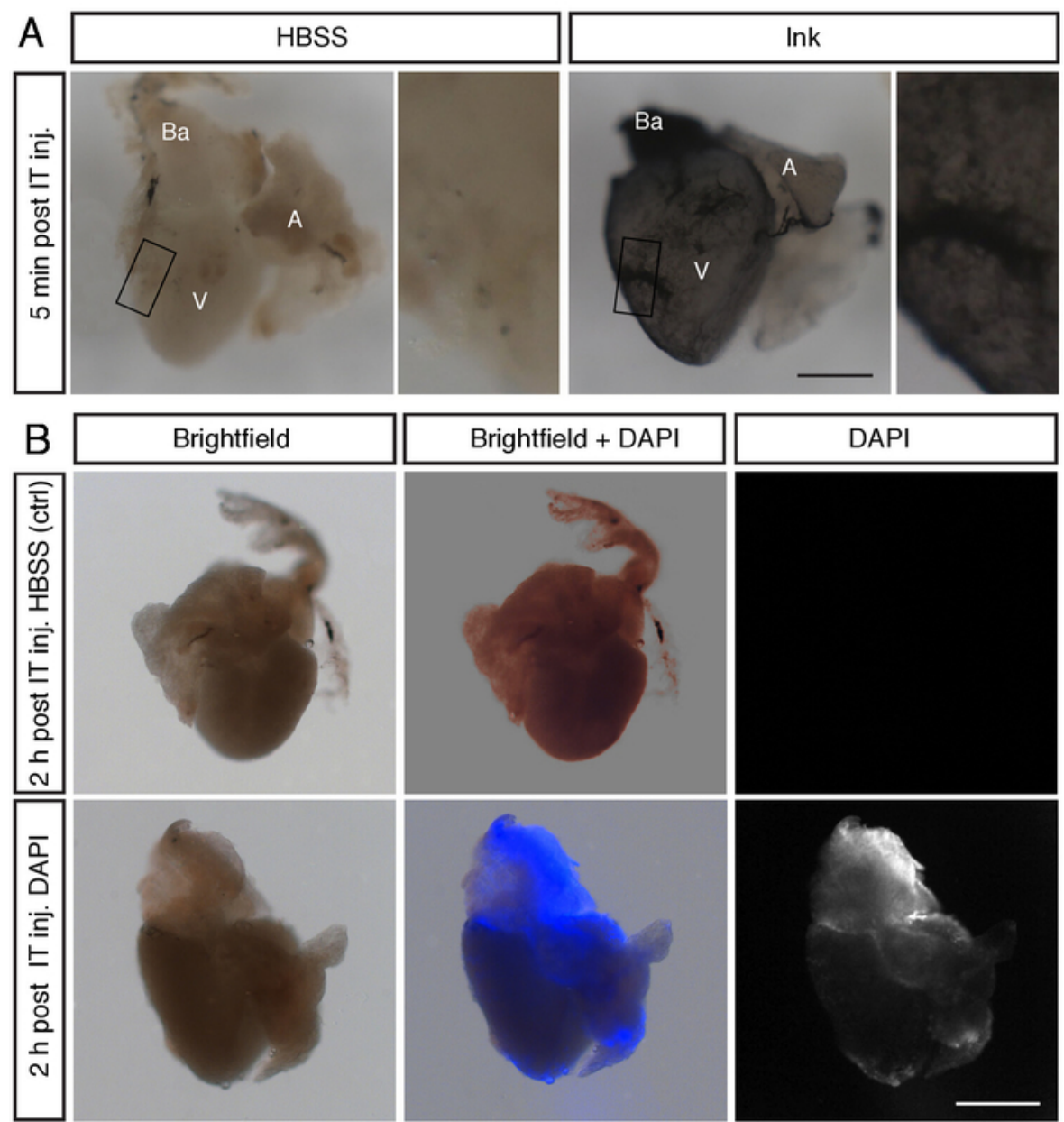

Figure 2: IT injected solutions spread nearly uniformly on the heart surface. (A) Stereomicroscope images of whole hearts of fish subjected to post-mortem IT injection with $2.5 \mu \mathrm{L} \mathrm{HBSS}$ or $2.5 \mu \mathrm{L}$ ink. Ink stained the surface of the ventricle $(\mathrm{V})$, atrium (A) and bulbus arteriosus (Ba). Scale bar $=300 \mu \mathrm{m}$. (B) Bright-field and fluorescent stereomicroscope images of whole hearts of fish subjected to IT injection with HBSS and $3 \mu \mathrm{L}$ of $1 \mu \mathrm{g} / \mathrm{mL}$ DAPI. DAPI fluorescence is detected on the heart parts soon after IT injection. Scale bar $=300 \mu \mathrm{m}$. Please click here to view a larger version of this figure. 

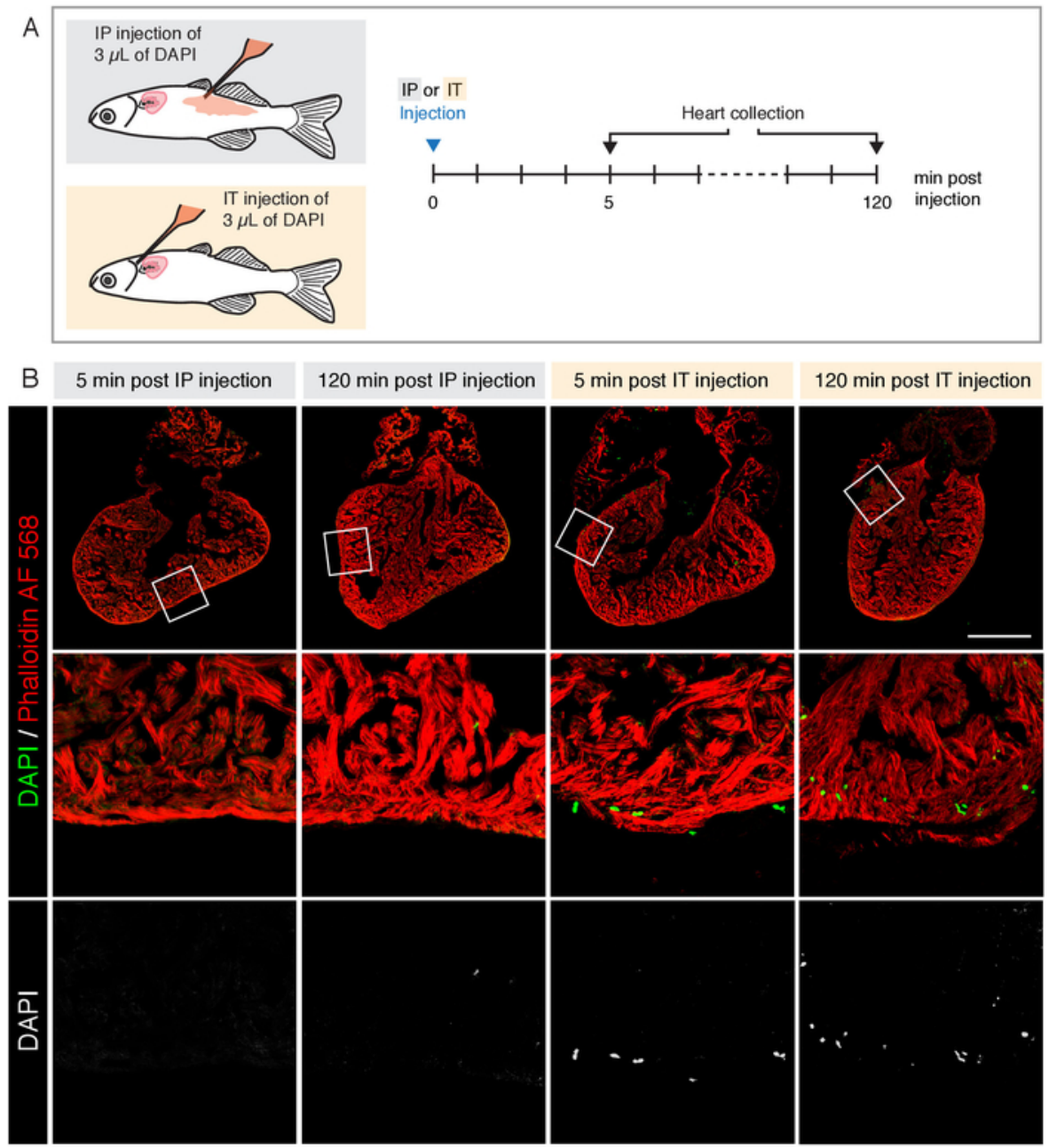

Figure 3: Comparison of two injection methods for the delivery of DAPI to the heart. (A) Scheme of the experimental design. Intraperitoneal (IP) and Intrathorascic (IT) injections were performed with the same amount of $1 \mu \mathrm{g} / \mathrm{mL}$ DAPI $(3 \mu \mathrm{L})$. Hearts were collected at 5 and 120 min after injection. (B) Confocal microscopy images of heart sections stained with fluorescent phalloidin (red) that abundantly labels muscle fibers. Injected DAPI was visualized in the appropriate channel shown in green. After IP injection, DAPI is not detected in the heart at any time point. After IT injection, DAPI positive cells are present in the ventricle after both time points. Scale bar $=500 \mu \mathrm{m}$. Please click here to view a larger version of this figure. 
A

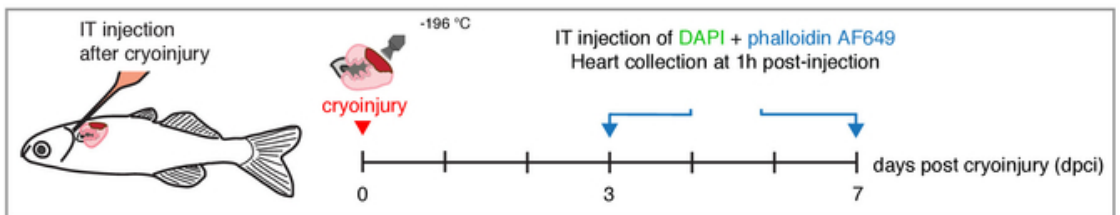

B
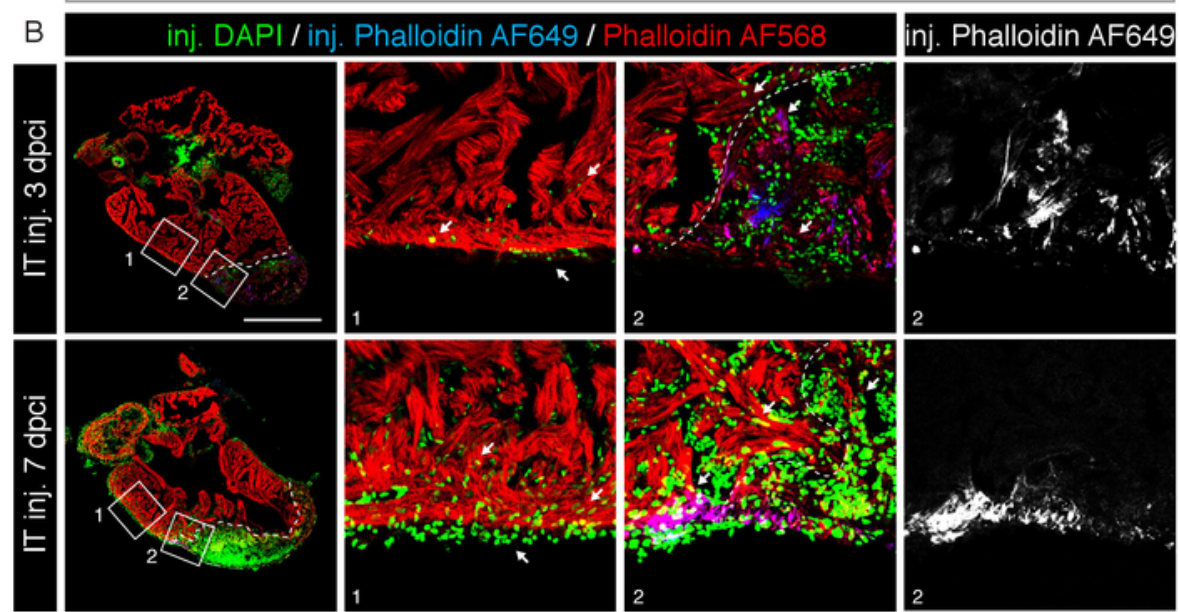

Figure 4: IT injection to study heart regeneration. (A) Scheme of the experimental design. At 3 and 7 days following cryoinjury, a mix of DAPI and phalloidin AF649 was IT-injected $(3 \mu \mathrm{L}$ of $1 \mu \mathrm{g} / \mathrm{mL})$. Hearts were collected 1 hour after IT injection, fixed, sectioned and stained with phalloidin AF568 (red). (B) Confocal microscopy images of longitudinal heart sections at 3 and 7 dpci. Injected DAPI (green) and phalloidin AF649 (blue) label cells of the injured area (delimited by white dashed line) and the intact myocardium (red staining). White arrows are pointing at DAPI (green) distribution through intact compact and trabeculated myocardia and the epicardium. Scale bar $=500 \mu \mathrm{m}$. Please click here to view a larger version of this figure. 
A
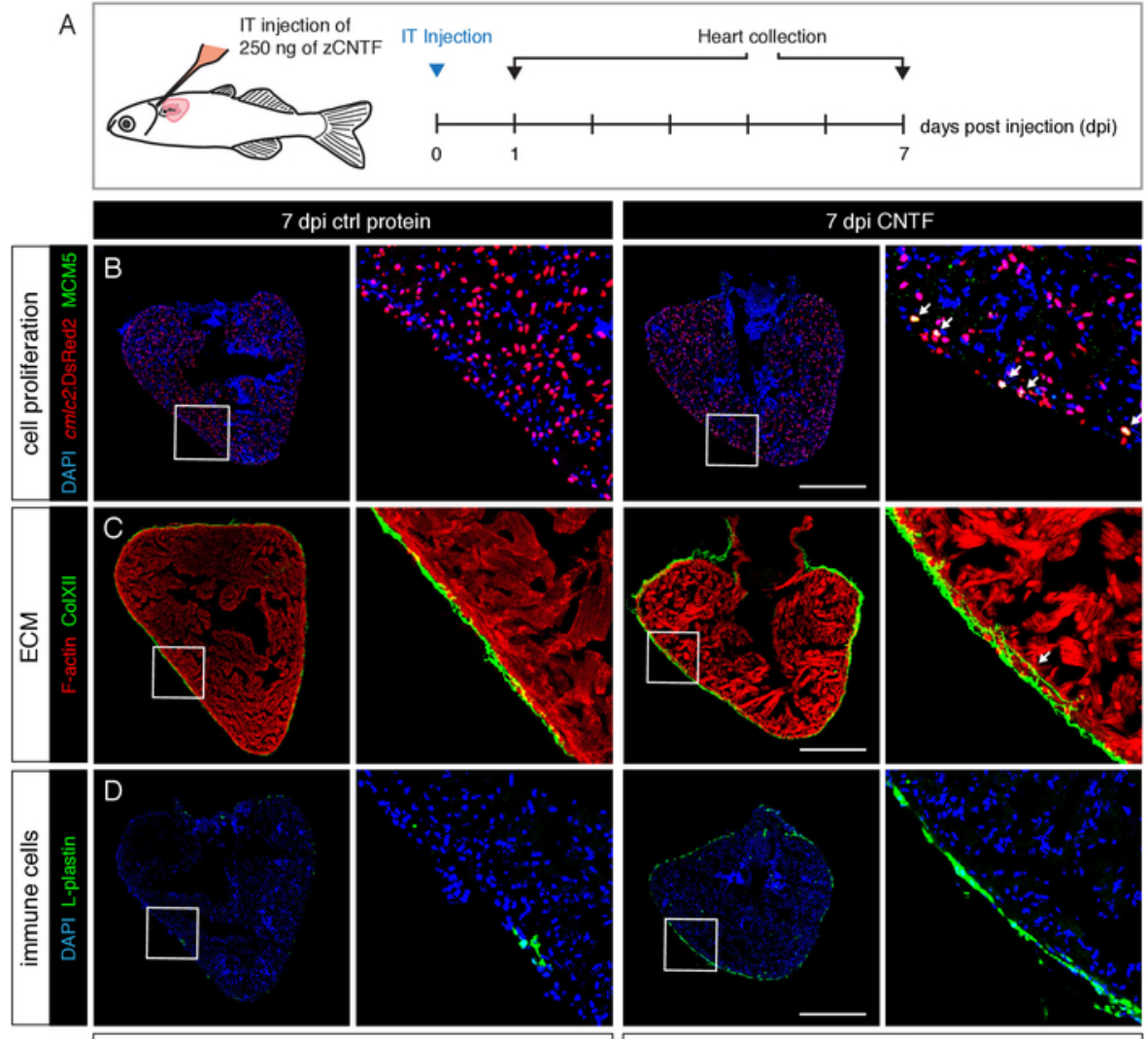

$\mathrm{E}$

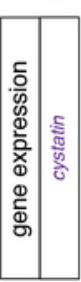

1 dpi hlgG
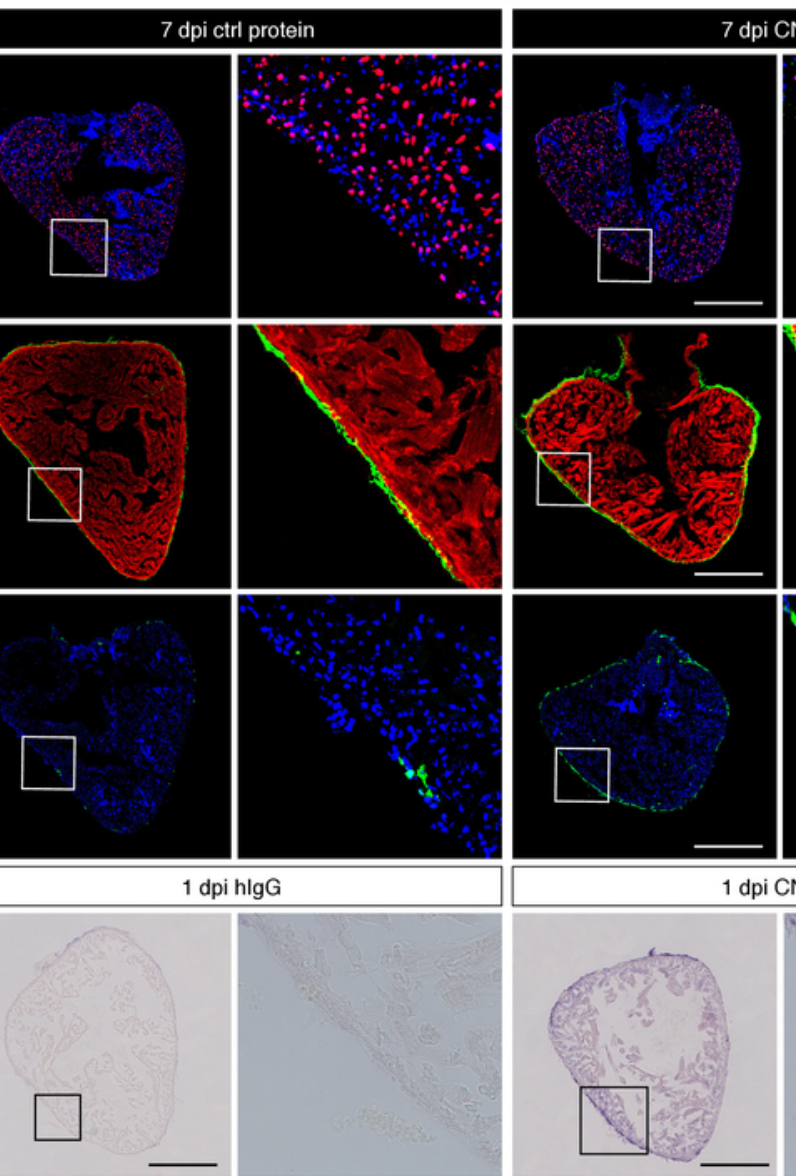

dpi CNTF

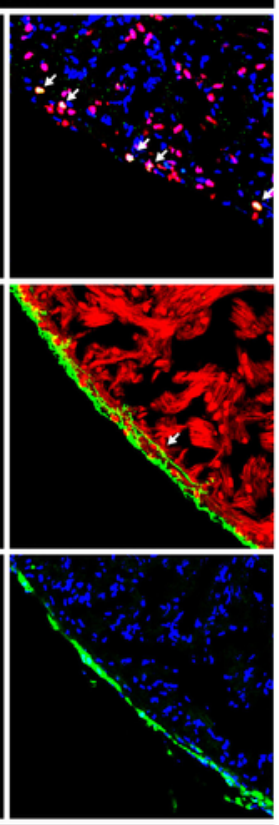

1 dpi CNTF

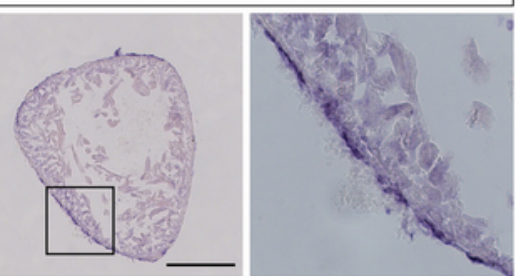

Figure 5: Exogenous IT-injected CNTF stimulates several biological processes in the heart. (A) Scheme of the experimental design. First, $2.5 \mu \mathrm{L}$ of a solution containing $250 \mathrm{ng}$ of zebrafish CNTF or control immunoglobulins (hlgG) was injected into the pericardium of transgenic fish expressing nuclear DsRed2 in cardiomyocytes. Hearts were collected at 7 and 1 days post-injection (dpi) and analyzed by immunofluorescence and in situ hybridization, respectively. (B-D) Confocal microscopy images of ventricular sections of control and CNTF-injected hearts. (B) Immunostaining against a cell cycle marker, minichromosome maintenance complex component 5 (MCM5; green), reveals a higher number of proliferating cardiomyocytes in response to exogenous CNTF. Scale bar $=500 \mu \mathrm{m}$. (C) Immunostaining against collagen XII shows increased deposition of collagen XII in the myocardium after CNTF injection. In control heart, collagen XII is confined to the epicardium ${ }^{34}$. Scale bar $=500$ $\mu \mathrm{m}$. (D) Immunostaining against an immune cell marker, L-plastin, detects an enhanced recruitment of immune cells in the CNTF injected fish. Scale bar $=500 \mu \mathrm{m}$. (E) Bright-field microscope images of ventricular cross sections after in situ hybridization using an antisense mRNA probe against cystatin, a cardioprotective factor, displays transcriptional upregulation of this gene in the heart of CNTF-injected fish. Scale bar $=500$ $\mu \mathrm{m}$. This figure has been modified from Bise et al. ${ }^{24}$. Please click here to view a larger version of this figure.

\section{Discussion}

Here, we describe a method for delivering exogenous compounds and proteins into the pericardial cavity in order to study their effects on the heart in adult zebrafish. The procedure is based on intrathoracic injection, which results in the delivery of a small volume of solution in the vicinity of the organ. This technique was developed and described for studying cardiac preconditioning and regeneration.

The critical step in this procedure is the penetration of the glass capillary into the thoracic cavity. This step depends on three parameters which are: the rigidity and sharpness of the capillary tip, the angle of penetration, and the puncture site. To optimize the penetration through the skin, the pulled part of the capillary should not to be too long, as such needles are too flexible and bend in contact with the skin. To avoid this, the rigidity can be adapted by reducing the tip size with the iridectomy scissor. Although the angle of penetration can vary between $30^{\circ}$ and $45^{\circ}$, it can be adapted to the rigidity of the tip. Indeed, a thin tip will penetrate the skin better with a narrower angle. 
In order to optimize the needle penetration, the insertion site should be immediately above the beating heart. The risk of heart puncture is usually low ranging between $5 \%$ and $8 \%$. Insertion of the needle posterior to the heart increases the risk of heart puncture, as seen by enhanced bleeding. In such cases, the animals should be removed from the experiments.

Another source of trouble during the IT injection occurs at the capillary level. Indeed the capillary can break when lateral forces are exerted on it. To avoid this, the needle must move along the axis of injection in a straight way. Occasionally, the capillary can be blocked by tissue residues that prevent the liquid from flowing. The needle can be unblocked by gently withdrawing the tip whilst injecting. If this does not improve the flow, we recommend completely withdrawing the needle from the thorax and replacing the needle.

Lesions can be caused by a too deeply inserted needle in the pericardium. In order to avoid lesions in the pericardial sac, the needle must not be inserted too much (1-2 mm) into the thorax. Some leaks were observed when the injection volume was bigger than $8 \mu \mathrm{L}$

In zebrafish, the exact composition of the pericardial fluid is unknown. However, the volume of the pericardial cavity is estimated at $\sim 10 \mu \mathrm{L}^{31}$ Given that the volume of the adult zebrafish ventricle is approximately $1-2 \mathrm{~mm}^{3}$, we assume that the pericardial cavity accordingly has a tiny volume, which has to be considered before injections. From our preliminary studies, we determined that the optimal range of the injected volume is between 0.5 and $3 \mu \mathrm{L}$ for fish measuring $2.5-2.8 \mathrm{~cm}$ (distance from the snout to the caudal peduncle). This volume can be adapted depending on the size of the fish. Injection of up to $5 \mu \mathrm{L}$ did not induce any lesion in the fish of this size. However, volumes from $8 \mu \mathrm{L}$ were sufficient to cause bulging and internal bleeding as shown in Figure 1F. Based on this data, we estimate that an amount of solution bigger than $3 \mu \mathrm{L}$ might cause physical and physiological stress on the organ. This limitation infers the need to choose a higher concentration of molecules instead of increasing the amount of the injected solution.

Another important factor is the osmotic property of the injected solution, which should be in the physiological range. Indeed, to avoid a risk of osmotic stress, we recommend HBSS as injection medium.

In zebrafish, the common methods used to deliver drugs are through water treatment and intraperitoneal injection ${ }^{30,35}$. Although both of these techniques are suitable for many applications, IT injections provide experimental and economic advantages, by decreasing the risks of undesired systemic side effects and reducing the usage of costly molecules, respectively. This method can be suitable for delivery of tamoxifen to activate the Cre-ERT2 transgenic system used for cell lineage tracing analysis, and guide modified RNAs for functional studies in regeneration research.

The IT-injection method in zebrafish has been previously described ${ }^{31,36}$. In those reports, intrathoracic injections were performed with insulin needle, puncturing from the anterior side. In contrast, our protocol presents an alternative strategy with the pulled glass capillary inserted from the posterior direction. Specifically, our approach takes into account the anatomy of the fish pericardium to optimize the injection with a reduced risk of heart puncture. Furthermore, during the procedure, the fish is not held by metallic forceps, but by a moist and soft sponge, which is a more suitable method to avoid any external injury of the fish. Thus, the presented method might be better suited for studies of cardiac homeostasis, preconditioning and regeneration in adult zebrafish.

IT injections have already been established in mammalian model organisms. Indeed, this method has also been applied in experiments with pigs and clinical studies in humans ${ }^{37,38}$. In mice, transthoracic intramyocardial injections guided by ultrasounds have been used to challenge their heart ${ }^{39}$. Within this article we propose a detailed protocol to ease the use of IT injection for zebrafish. This will be particularly valuable to the field, in order to complement genetic approaches in cardiac homeostasis, preconditioning and regeneration research.

\section{Disclosures}

The authors have nothing to disclose.

\section{Acknowledgments}

We thank V. Zimmermann for excellent technical assistance and for fish care, D. König (University of Fribourg) for critical reading of the manuscript, D. Kressler (University of Fribourg) for help with zCNTF protein synthesis, F. Ruggiero (Institut de Génomique Fonctionnelle de Lyon) for providing ColXII antibody, and P. Martin (University of Bristol) for L-plastin antibody. We thank the imaging core facility and the proteomics platform at the University of Fribourg. This work was supported by the Swiss National Science Foundation, grant number 310030_179213, and by the Schweizerische Herzstiftung (Swiss Heart Foundation).

1. Tzahor, E., Poss, K. D. Cardiac regeneration strategies: Staying young at heart. Science. 356 (6342), 1035-1039 (2017).

2. lismaa, S. E. et al. Comparative regenerative mechanisms across different mammalian tissues. npj Regenerative Medicine. 3 (1) (2018).

3. Xiang, M. S. W., Kikuchi, K. Endogenous Mechanisms of Cardiac Regeneration. Int Rev Cell Mol Biol. 326, 67-131 (2016).

4. González-Rosa, J. M., Burns, C. E., Burns, C. G. Zebrafish heart regeneration: 15 years of discoveries. Regeneration. 4 (3), 105-123 (2017).

5. Jazwinska, A., Sallin, P. Regeneration versus scarring in vertebrate appendages and heart. The Journal of Pathology. 238 (2), $233-246$ (2016).

6. Sehring, I. M., Jahn, C., Weidinger, G. Zebrafish fin and heart: what's special about regeneration? Current Opinion in Genetics \& Development. 40, 48-56 (2016).

7. Rubin, N., Harrison, M. R., Krainock, M., Kim, R., Lien, C. L. Recent advancements in understanding endogenous heart regeneration-insights from adult zebrafish and neonatal mice. Seminars in Cell and Developmental Biology. 58, 34-40 (2016).

8. Chablais, F., Veit, J., Rainer, G., Jazwinska, A. The zebrafish heart regenerates after cryoinjury-induced myocardial infarction. BMC Developmental Biology. 11, 21 (2011). 
9. Schnabel, K., Wu, C. C., Kurth, T., Weidinger, G. Regeneration of cryoinjury induced necrotic heart lesions in zebrafish is associated with epicardial activation and cardiomyocyte proliferation. PLoS One. 6 (4), e18503 (2011).

10. Gonzalez-Rosa, J. M., Martin, V., Peralta, M., Torres, M., Mercader, N. Extensive scar formation and regression during heart regeneration after cryoinjury in zebrafish. Development. 138 (9), 1663-1674 (2011).

11. Poss, K. D., Wilson, L. G., Keating, M. T. Heart regeneration in zebrafish. Science. 298 (5601), 2188-2190 (2002).

12. Cao, J., Poss, K. D. The epicardium as a hub for heart regeneration. Nature Reviews Cardiology. 15 (10), $631-647$ (2018).

13. Andres-Delgado, L., Mercader, N. Interplay between cardiac function and heart development. Biochim Biophys Acta. 1863 (7 Pt B), 1707-1716 (2016).

14. Richardson, R. J. Parallels between vertebrate cardiac and cutaneous wound healing and regeneration. npj Regenerative Medicine. 3, 21 (2018).

15. Lai, S.-L., Marín-Juez, R., Stainier, D. Y. R. Immune responses in cardiac repair and regeneration: a comparative point of view. Cellular and Molecular Life Sciences. (2018).

16. Kikuchi, K. et al. Primary contribution to zebrafish heart regeneration by gata4(+) cardiomyocytes. Nature. 464 (7288), 601-605 (2010).

17. Jopling, C. et al. Zebrafish heart regeneration occurs by cardiomyocyte dedifferentiation and proliferation. Nature. 464 (7288), 606-609 (2010).

18. Pfefferli, C., Jaźwińska, A. The careg element reveals a common regulation of regeneration in the zebrafish myocardium and fin. Nature Communications. 8, 15151 (2017).

19. Sánchez-Iranzo, H. et al. Tbx5a lineage tracing shows cardiomyocyte plasticity during zebrafish heart regeneration. Nature Communications. 9 (1) (2018).

20. Wang, J., Poss, K. D. Methodologies for Inducing Cardiac Injury and Assaying Regeneration in Adult Zebrafish. Methods In Molecular Medicine. 1451, 225-235 (2016).

21. Gut, P., Reischauer, S., Stainier, D. Y. R., Arnaout, R. Little Fish, Big Data: Zebrafish as a Model for Cardiovascular and Metabolic Disease. Physiological Reviews. 97 (3), 889-938 (2017).

22. de Preux Charles, A. S., Bise, T., Baier, F., Marro, J., Jazwinska, A. Distinct effects of inflammation on preconditioning and regeneration of the adult zebrafish heart. Open Biology. 6 (7) (2016).

23. de Preux Charles, A. S., Bise, T., Baier, F., Sallin, P., Jazwinska, A. Preconditioning boosts regenerative programmes in the adult zebrafish heart. Open Biology. 6 (7) (2016).

24. Bise, T., de Preux Charles, A. S., Jazwinska, A. Ciliary neurotrophic factor stimulates cardioprotection and the proliferative activity in the zebrafish adult heart. npj Regenerative Medicine. 4, (2019).

25. Thorimbert, V., Konig, D., Marro, J., Ruggiero, F., Jazwinska, A. Bone morphogenetic protein signaling promotes morphogenesis of blood vessels, wound epidermis, and actinotrichia during fin regeneration in zebrafish. The FASEB Journal. 29 (10), 4299-4312 (2015).

26. König, D., Page, L., Chassot, B., Jaźwińska, A. Dynamics of actinotrichia regeneration in the adult zebrafish fin. Developmental Biology. 433 (2), 416-432 (2018).

27. Sallin, P., Jaźwińska, A. Acute stress is detrimental to heart regeneration in zebrafish. Open Biology. 6 (3), 160012 (2016).

28. Mokalled, M. H., Poss, K. D. A Regeneration Toolkit. Developmental Cell. 47 (3), 267-280 (2018).

29. Pugach, E. K., Li, P., White, R., Zon, L. Retro-orbital Injection in Adult Zebrafish. Journal of Visualized Experiments. (34), e1645 (2009).

30. Kinkel, M. D., Eames, S. C., Philipson, L. H., Prince, V. E. Intraperitoneal Injection into Adult Zebrafish. Journal of Visualized Experiments. (42) (2010)

31. Xiao, C. et al. Nanoparticle-mediated siRNA Gene-silencing in Adult Zebrafish Heart. Journal of Visualized Experiments. (137) (2018).

32. Chablais, F., Jazwinska, A. Induction of myocardial infarction in adult zebrafish using cryoinjury. Journal of Visualized Experiments. (62) (2012).

33. Gonzalez-Rosa, J. M., Mercader, N. Cryoinjury as a myocardial infarction model for the study of cardiac regeneration in the zebrafish. Nature Protocols. 7 (4), 782-788 (2012)

34. Marro, J., Pfefferli, C., de Preux Charles, A. S., Bise, T., Jazwinska, A. Collagen XII Contributes to Epicardial and Connective Tissues in the Zebrafish Heart during Ontogenesis and Regeneration. PLoS One. 11 (10), e0165497 (2016).

35. Ma, X., Ding, Y., Wang, Y., Xu, X. A Doxorubicin-induced Cardiomyopathy Model in Adult Zebrafish. Journal of Visualized Experiments. (136) (2018).

36. Diao, J. et al. PEG-PLA nanoparticles facilitate siRNA knockdown in adult zebrafish heart. Developmental Biology. 406 (2), $196-202$ (2015).

37. Lloyd, L. C., Etheridge, J. R. The pathological and serological response induced in pigs by parenteral inoculation of Mycoplasma hyopneumoniae. Journal of Comparative Pathology. 91 (1), 77-83 (1981).

38. Zhou, A., Guo, L., Tang, L. Effect of an intrathoracic injection of sodium hyaluronic acid on the prevention of pleural thickening in excess fluid of tuberculous thoracic cavity. Clinical and Experimental Pharmacology and Physiology. 30 (3), 203-205 (2003).

39. Prendiville, T. W. et al. Ultrasound-guided Transthoracic Intramyocardial Injection in Mice. Journal of Visualized Experiments. (90) (2014). 